\title{
Drell-Yan production at NLO in the Parton Branching method at low and high DY masses
}

\author{
Qun Wang, ${ }^{a, b, *}$ Armando Bermudez Martinez, ${ }^{b}$ Patrick Connor, ${ }^{b}$ Luis Ignacio Estevez \\ Banos, ${ }^{b}$ Francesco Hautmann, ${ }^{c, d}$ Hannes Jung, ${ }^{b}$ Jindrich Lidrych, ${ }^{b}$ Aleksandra \\ Anna Lelek, ${ }^{d}$ Melanie Viola Schmitz, ${ }^{b}$ Sara Taheri Monfared, ${ }^{b}$ Thomas Wening $^{b}$ and \\ Heng Yang ${ }^{a, b}$ \\ ${ }^{a}$ School of Physics, Peking University, \\ Yiheyuan road 5, Haidian District, Beijing, China \\ ${ }^{b}$ Deutsches Elektronen-Synchrotron, D-22607 Hamburg, Germany \\ ${ }^{c}$ RAL, Chilton OX11 OQX and University of Oxford, OX1 3NP \\ ${ }^{d}$ University of Antwerp, Antwerp, Belgium \\ E-mail: qun.wang@desy.de, hannes.jung@desy.de
}

It has been observed that measurements of low-mass Drell-Yan (DY) transverse momentum spectra at low center-of-mass energies $\sqrt{s}$ are not well described by perturbative QCD calculations in collinear factorization in the region where transverse momenta are comparable with the DY mass. This issue can be examined with the Parton Branching (PB) method combining next-to-leadingorder (NLO) calculations of the hard process with the evolution of transverse momentum dependent (TMD) parton distribution. The predictions are compared with measurements of low mass DY production, and they are in very good agreement. Predictions have also been compared with the measurement of DY production at high energies at the LHC. We find that at low-mass DY and low $\sqrt{s}$ even in the region of $p_{T} / m_{D Y} \sim 1$ the contribution of multiple soft gluon emissions (included in the PB-TMDs) is essential to describe the measurements, while at larger masses ( $m_{D Y} \sim m_{Z}$ ) and LHC energies the contribution from soft gluons in the region of $p_{T} / m_{D Y} \sim 1$ is small.

40th International Conference on High Energy physics - ICHEP2020

July 28 - August 6, 2020

Prague, Czech Republic (virtual meeting)

\footnotetext{
*Speaker
} 
The production of Z-bosons in a Drell-Yan (DY) [1] in pp collisions is one of the most precisely measured processes at the LHC [2-4, 8, 9]. To describe the measurement of DY production, higher-order calculations in perturbative QCD are necessary. At small transverse momenta $\left(p_{T}<\right.$ $O\left(m_{Z}\right)$ ) the production of Z-bosons cannot be described by fixed order calculations, and soft gluon resummation to all orders and nonperturbative contributions are needed.

Soft gluon resummation in low $p_{T}$ spectrum can be achieved by analytic TMD resummation methods or by parton shower in multi-purpose Monte Carlo (MC) event generators matched with higher-order matrix elements. Nonperturbative effects can be incorporated via transverse momentum dependent parton distribution functions (TMDs) at low transverse momenta.

Z-boson $p_{T}$ spectrum can also be accurately evaluated by using the Parton Branching (PB) formulation of TMD evolution [5-7] together with NLO calculations of the hard scattering process in the MADGRAPH5_AMC@NLO framework. The obtained predictions were found to be in very good agreement with measurements from ATLAS at $\sqrt{s}=8 \mathrm{TeV}$ [3], and CMS at $\sqrt{s}=13 \mathrm{TeV}$ [9], with modest sensitivity to the nonperturbative part of the TMD distributions.

At lower mass $m_{D Y}$ the DY process is experimentally challenging, especially at LHC. At lower center-of-mass energies DY measurements covering the low $p_{T}$ region exist from PHENIX [10] at $\sqrt{s}=200 \mathrm{GeV}$, from R209 [11] at $\sqrt{s}=62 \mathrm{GeV}$, and from NuSea [12, 13] at $\sqrt{s}=38.8 \mathrm{GeV}$. The predictions for low mass and low $\sqrt{s}$ are obtained using the PB method for the TMD parton desities together with an NLO calculation of DY production. These low energy measurements are very well described with the PB-MC@NLO approach in the whole region of $p_{T} / m_{D Y}$.

\section{DY production at the LHC}

We make use of MADGRAPH5_AMC@NLO (version 2.6.4, in the following labelled MC@NLO) framework and apply the NLO PB parton distributions with $\alpha_{s}\left(M_{Z}\right)=0.118$ for the NLO calculations of inclusive Drell-Yan production. The transverse momenta according to the PB TMDs are used to modify the kinematics of the initial state parton (and as a consequence the final state partons and particles).

\subsection{Transverse momentum of $\mathrm{Z}$ production at $8 \mathrm{TeV}$}

The prediction for the transverse momentum spectrum of Z-bosons at $\sqrt{s}=8 \mathrm{TeV}$ are obtained using MC@NLO together with the PB-TMD, and it is compared with the measurement by ATLAS [3], as shown in figure 1. The PB-2018-Set2 parton distributions are used for both the collinear and the TMD calculations. The uncertainties coming from experimental and model uncertainties are shown, they are very small $(\sim 2 \%)$. The uncertainties coming from the scale variation ( $\mu_{F}$ and $\mu_{R}$ are varied by a factor of 2 up and down independently) are also shown in figure 1 . The prediction agrees very well with the measurement. The deviation of the prediction at higher transverse momenta comes from missing higher order contributions in the matrix element calculations as only $O\left(\alpha_{s}\right)$ corrections are included.

The contribution from DY +1 jets (with $p_{T}^{j e t}>10 \mathrm{GeV}$ ) at NLO is also shown in figure 1 . The higher order contribution plays an important role at larger $p_{T}$ and improves the description of the measurements. 

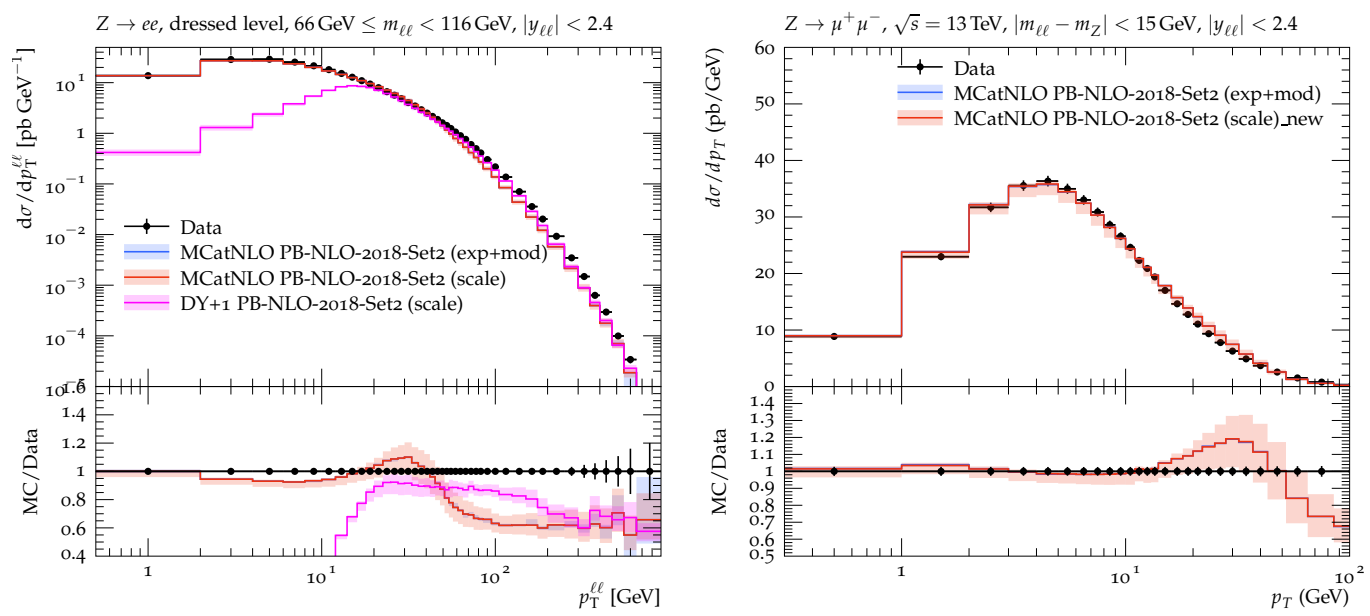

Figure 1: On the left (right) transverse momentum $p_{T}$ spectrum of Z-boson as measured by ATLAS at 8 TeV (CMS at $13 \mathrm{TeV}$ ) compared to the prediction from MC@NLO with PB-TMD NLO 2018 with uncertainties from the TMDs and scale variation combined.

\subsection{Transverse momentum at $13 \mathrm{TeV}$}

We obtained also predictions for the transverse momentum spectrum of Z-bosons at $\sqrt{s}=13$ $\mathrm{TeV}$ by CMS [9] using the same method as above. In figure 1 the prediction is compared with a measurement of CMS. As discussed in Ref. [14], the drop at large transverse momenta region in the prediction comes from missing NLO contribution to $\mathrm{Z}+$ jet production, i.e., $O\left(\alpha_{s}\right)$ terms in the hard process calculation.

\section{Low mass DY production}

We evaluate DY spectra at low DY masses [15] with PB-TMDs with MC@NLO.

\subsection{DY mass spectrum at low $\sqrt{s}$}

We start with the DY mass spectrum at low masses and low $\sqrt{s}$. Theoretical predictions of the DY mass spectrum obtained from PB-TMDs and NLO matrix elements using MC@NLO are compared in figure 2 with experimental measurements for different center-of-mass energies from NuSea [12, 13], R209 [11], and PHENIX [10]. The uncertainties coming from the determination of the PB-TMDs as well as from the variation of the scale in the perturbative calculation are shown as band. As already observed for the case of Z-production at the LHC, the contribution to uncertainties from the parton density turns out to be small compared to the one from the scale uncertainty.

In the figure 2, the DY mass spectra are generally well described by the PB-TMDs + NLO calculation. For the region of highest masses at lowest $\sqrt{s}$ from NuSea, the description of experimental data by PB-TMDs + NLO calculation deteriorates. This is because the parton densities used in the calculation, which are determined from fits to HERA data, are poorly constrained in the large- $x$ region. The description in this region can be readily improved by using parton density sets from global fits, NNPDF3.0 [? ]. On the other hand, the mass spectrum is well described for the lowest mass region $m_{D Y}<6 \mathrm{GeV}$ of NuSea. 

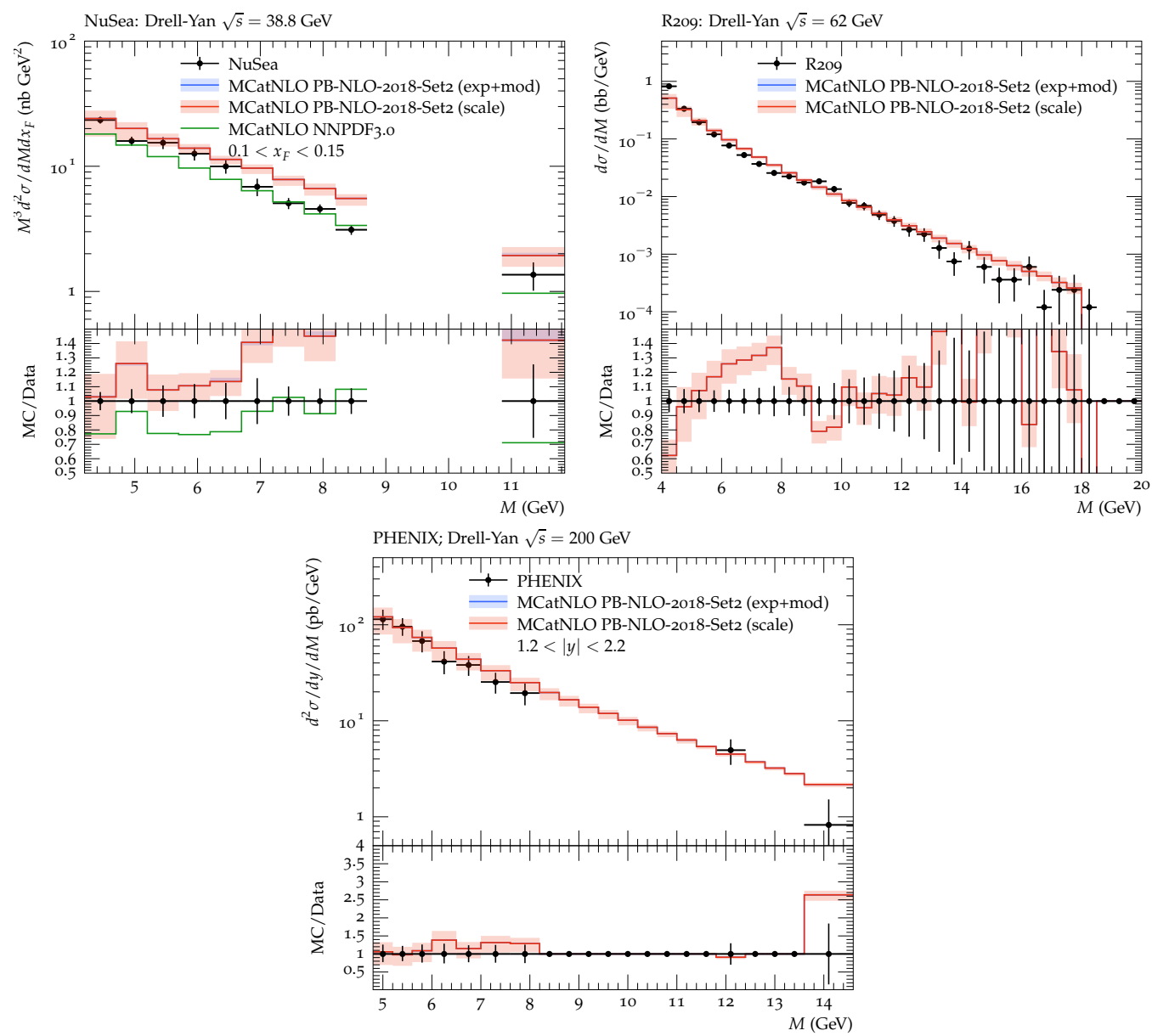

Figure 2: Drell-Yan mass distribution production measured by NuSea, R209, and PHENIX compared to predictions at NLO using PB-TMDs. For NuSea, the prediction using NNPDF3.0 is also shown.

\subsection{DY transverse momentum spectra}

We compare in figure 3 predictions obtained with PB-TMDs and NLO matrix using MC@ NLO for the transverse momentum spectra of DY with experimental measurements for different center-ofmass energies from NuSea, R209 and PHENIX. The quality of the description of the measurements (including independent variations of the factorization and renormalization scales by a factor of two up and down) is good with $\chi^{2} / n d f=1.08,1.27,1.04$ for NuSea, R209 and PHENIX, respectively. The $\chi^{2}$ values are calculated using the full $p_{T}$ range.

It has been observed in [16] that fixed-order calculations at $O\left(\alpha_{s}\right)$ and $O\left(\alpha_{s}^{2}\right)$ in collinear factorization are not able to describe the DY transverse momentum spectra at low $\sqrt{s}$ in the region $p_{T} / m_{D Y} \sim 1$. This indicates that the inclusion of multiple parton emissions, taken into account by the PB-TMD, is essential to describe the region $p_{T} / m_{D Y} \sim 1$ at low energies. 

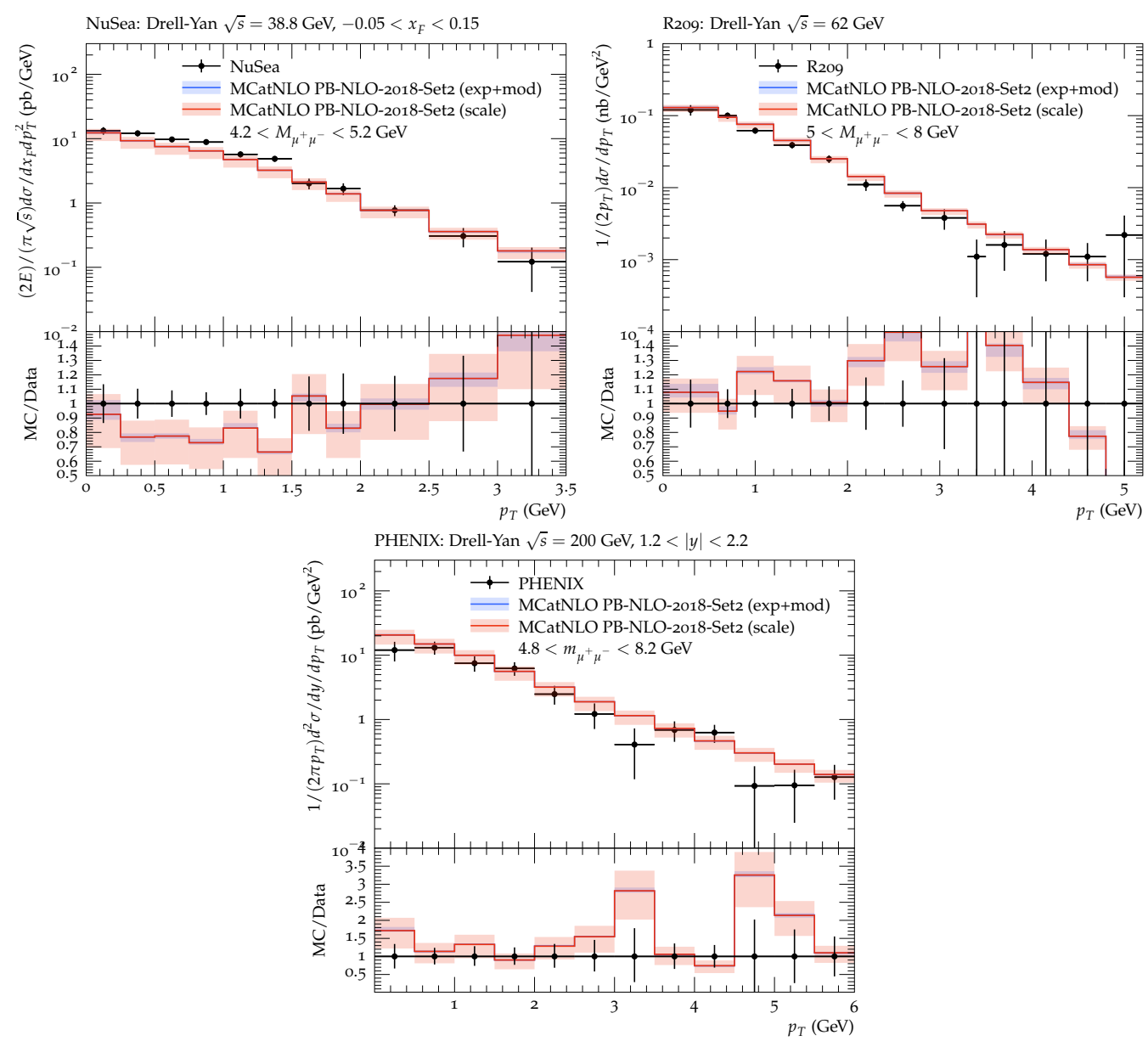

Figure 3: Drell-Yan mass distribution production measured by NuSea, R209, and PHENIX compared to predictions at NLO using PB-TMDs. For NuSea, the prediction using NNPDF3.0 is also shown.

\section{Conclusion}

We have presented predictions for the transverse momentum spectra of DY production at high center-of-mass energies as well as at low center-of-mass energies obtained from the PB-TMD distributions and NLO calculations via MC@NLO. The predictions describes well the measurements at the LHC. We also observe a very good description of the measurements of NuSea at $\sqrt{s}=38$ $\mathrm{GeV}, \mathrm{R} 209$ at $\sqrt{s}=62 \mathrm{GeV}$, and PHENIX at $\sqrt{s}=200 \mathrm{GeV}$, with values of $\chi^{2} / n d f \sim 1$ for all the measurements. We notice that, at low DY mass and low $\sqrt{s}$, even in the region of $p_{T} / m_{D Y} \sim 1$ the contribution of QCD multi-parton radiation is essential to describe the measurements, while at larger masses $\left(m_{D Y} \sim m_{Z}\right)$ and LHC energies this contribution is small in the region of $p_{T} / m_{D Y} \sim 1$.

\section{References}

[1] S. Drell and T.-M. Yan, "Massive Lepton Pair Production in Hadron-Hadron Collisions at High-Energies", Phys.Rev.Lett. 25 (1970) 316-320. 
[2] CMS Collaboration, "Measurement of the transverse momentum spectra of weak vector bosons produced in proton-proton collisions at $\sqrt{s}=8 \mathrm{TeV}$ ", JHEP 02 (2017) 096, arXiv: 1606.05864.

[3] ATLAS Collaboration, "Measurement of the transverse momentum and $\phi_{\eta}^{*}$ distributions of Drell-Yan lepton pairs in proton-proton collisions at $\sqrt{s}=8 \mathrm{TeV}$ with the ATLAS detector", Eur. Phys. J. C76 (2016), no. 5, 291, arXiv: 1512 . 02192.

[4] CMS Collaboration, "Measurement of the rapidity and transverse momentum distributions of $Z$ Bosons in pp collisions at $\sqrt{s}=7$ TeV”, Phys.Rev. D85 (2012) 032002, arXiv: 1110 . 4973.

[5] A. Bermudez Martinez, P. Connor, F. Hautmann, H. Jung, A. Lelek, V. Radescu et al., Collinear and TMD parton densities from fits to precision DIS measurements in the parton branching method, Phys. Rev. D99 (2019) 074008 [1804.11152].

[6] F. Hautmann, H. Jung, A. Lelek, V. Radescu and R. Zlebcik, Collinear and TMD quark and gluon densities from Parton Branching solution of QCD evolution equations, JHEP 01 (2018) 070 [1708.03279].

[7] F. Hautmann, H. Jung, A. Lelek, V. Radescu and R. Zlebcik, Soft-gluon resolution scale in QCD evolution equations, Phys. Lett. B772 (2017) 446 [1704 . 01757].

[8] ATLAS collaboration, Measurement of the transverse momentum distribution of Drell-Yan lepton pairs in proton-proton collisions at $\sqrt{s}=13 \mathrm{TeV}$ with the ATLAS detector, 1912.02844.

[9] CMS collaboration, Measurements of differential Z boson production cross sections in protonproton collisions at $\sqrt{s}=13 \mathrm{TeV}, \mathrm{JHEP} 12$ (2019) 061 [1909.04133].

[10] PHENIX collaboration, Measurements of $\mu \mu$ pairs from open heavy flavor and Drell-Yan in $p+p$ collisions at $\sqrt{s}=200$ GeV, Phys. Rev. D99 (2019) 072003 [1805. 02448].

[11] D. Antreasyan et al., Dimuon Scaling Comparison at 44-GeV and 62-GeV, Phys. Rev. Lett. 48 (1982) 302.

[12] NuSEa collaboration, Absolute Drell-Yan dimuon cross sections in 800-GeV/c p p and $p d$ collisions, hep-ex/0302019.

[13] J. C. Webb, Measurement of continuum dimuon production in 800-GeV/c proton nucleon collisions, hep-ex/0301031.

[14] A. Bermudez Martinez et al., Production of Z-bosons in the parton branching method, Phys. Rev. D100 (2019) 074027 [1906.00919].

[15] A. Bermudez Martinez et al., The transverse momentum spectrum of low mass Drell-Yan production at next-to-leading order in the parton branching method, Eur. Phys. J. C 80 (2020) 598, arXiv: 2001.06488.

[16] A. Bacchetta, G. Bozzi, M. Lambertsen, F. Piacenza, J. Steiglechner and W. Vogelsang, Difficulties in the description of Drell-Yan processes at moderate invariant mass and high transverse momentum, Phys. Rev. D100 (2019) 014018 [1901.06916]. 